POS $\quad$ PROCEEDINGS

\title{
AGB Evolution and Nucleosynthesis at Low-Metallicity Constrained by the Star Formation History of Our Galaxy
}

\section{Takuma Suda*}

National Observatory of Japan, Osawa 2-21-1, Mitaka, Tokyo, 181-8588, Japan

E-mail: takuma.suda@nao.ac.jp

\section{Yutaka Komiya}

National Astronomical Observatory of Japan, Osawa 2-21-1, Mitaka, Tokyo, 181-8588, Japan

\section{Shimako Yamada}

Hokkaido University, Kita 10 Nishi 8, Kita-ku, Sapporo, 060-0810, Japan

\section{Yutaka Katsuta}

Hokkaido University, Kita 10 Nishi 8, Kita-ku, Sapporo, 060-0810, Japan

\section{Wako Aoki}

National Astronomical Observatory of Japan, Osawa 2-21-1, Mitaka, Tokyo, 181-8588, Japan

\section{Pilar Gil-Pons}

Universitat Politecnica de Catalunya, Campus Baix Llobregat, Building C3, 08860 Castellefels, Spain

\section{Carolyn L. Doherty}

Monash University, Victoria 3800, Australia

\section{Simon W. Campbell}

Monash University, Victoria 3800, Australia

\section{Peter R. Wood}

Australian National University, Cotter Road, Weston Creek ACT 2611, Australia

\section{Masayuki Y. Fujimoto}

Hokkaido University, Kita 10 Nishi 8, Kita-ku, Sapporo, 060-0810, Japan 
We construct a binary population synthesis model to constrain the AGB evolution models from the star formation history of the Galaxy through the comparisons of model stars with observed extremely metal-poor stars. The binary population model includes the effects of AGB evolution and binary mass transfer for a given IMF and binary period distribution function. We discuss the origins of extremely metal-poor stars with enhancement of carbon or nitrogen, with the possible effect of mass loss at low-metallicity taken into account. Our results strongly support high-mass dominated star formation during the early epoch of the Galaxy in order to explain the observed frequency of carbon and nitrogen enhancements that are thought to result from mass transfer from a former AGB binary companion. Our model also favours the suppression of mass loss at lowmetallicity to reduce the number of the possible progenitors of nitrogen-enhanced stars when we adopt a massive star dominated IMF.

XII International Symposium on Nuclei in the Cosmos,

August 5-12, 2012

Cairns, Australia

* Speaker. 


\section{Introduction}

Extremely metal-poor $($ EMP) stars (defined by $[\mathrm{Fe} / \mathrm{H}]<-2.5$ ) in the Galaxy can help to constrain the evolution of low-metallicity stars. Many observations have revealed a large frequency of carbon-enhanced stars among EMP stars (CEMP stars)[1]. It is also known that there is a subclass of CEMP star that does not show any enhancement of s-process elements (CEMP-no stars), which apparently contradicts the expectation of the binary pollution scenario whereby observed stars change their surface abundances by mass transfer from AGB stars with enhanced abundances of carbon and s-process elements (corresponding to CEMP-s stars) [2]. The apparent lack of nitrogen-enhanced EMP stars (NEMP stars) is also a problem because intermediate-mass AGB stars will contribute to the EMP population through binary mass transfer after $\mathrm{CNO}$ cycling during hot bottom burning, which occurs during the thermal pulsing phase.

In this paper, we explore the constraints on the evolution of EMP AGB stars using binary population synthesis. Our model includes the effect of AGB evolution and binary mass transfer for a given Initial Mass Function (IMF) and period distribution function of binaries. In particular, we emphasise the role of the suppression of mass loss at low-metallicity [3] because it significantly influences the yields of EMP AGB stars. It is currently believed that the IMF for the first stars dominates high-mass stars, which is quite different from the characteristics prescribed by the present-day IMF[4]. Our previous works suggested that there was a transition of the IMF in the early Galaxy[5] 6$]$.

\section{Models and assumptions}

We modelled the stochastic formation of binary systems subject to the IMF, mass ratio function, and binary period distribution function using Monte-Carlo approach. Simulations are performed for each metallicity, from $[\mathrm{Fe} / \mathrm{H}]=-6$ to -1 in 0.5 dex steps. The model parameters for the binary population are the same as in [5] while the IMF is a log-normal distribution with a peak at $10 M_{\odot}$ and dispersion of 0.4 or a power law distribution with slopes of $x=0,-0.35,-0.85$, and -1.35 . The mass ratio function is taken from the data for nearby main sequence stars $[7,8]$. Period distribution functions are also taken from the literature [7,8,9]. The distribution of these quantities are shown in Figure 2

AGB yields are taken from the model calculations to cover the entire mass and metallicity range [10, 11] 12]. Binary mass transfer by wind accretion or Roche-Lobe overflow is followed depending on the masses and separation of the system according to [13 14]. For wind accretion, we assumed instantaneous mass transfer at the end of the dredge-up of carbon by the helium-flash driven deep mixing (He-FDDM) [15] 16] or the third dredge-up in the last model.

In our models, the mass and metallicity ranges of the progenitors for CEMP-s, CEMP-no, and NEMP stars are treated as parameters based on results of stellar evolution. For the formation of CEMP-s, the He-FDDM at $[\mathrm{Fe} / \mathrm{H}] \leq-2.5$ and the third dredge-up at $[\mathrm{Fe} / \mathrm{H}]>-2.5$ are assumed to be responsible, while the third dredge-up is assumed to be the source of CEMP-no at $[\mathrm{Fe} / \mathrm{H}] \leq-2.5$. NEMP stars are produced by the hot bottom burning at any metallicity range, while it is suppressed by the effect of mass loss at $[\mathrm{Fe} / \mathrm{H}] \leq-2.5$. In the fiducial model, we set the lower boundary for the third dredge-up and hot bottom burning at $1.5 M_{\odot}$ and $4.5 M_{\odot}$, respectively, inde- 

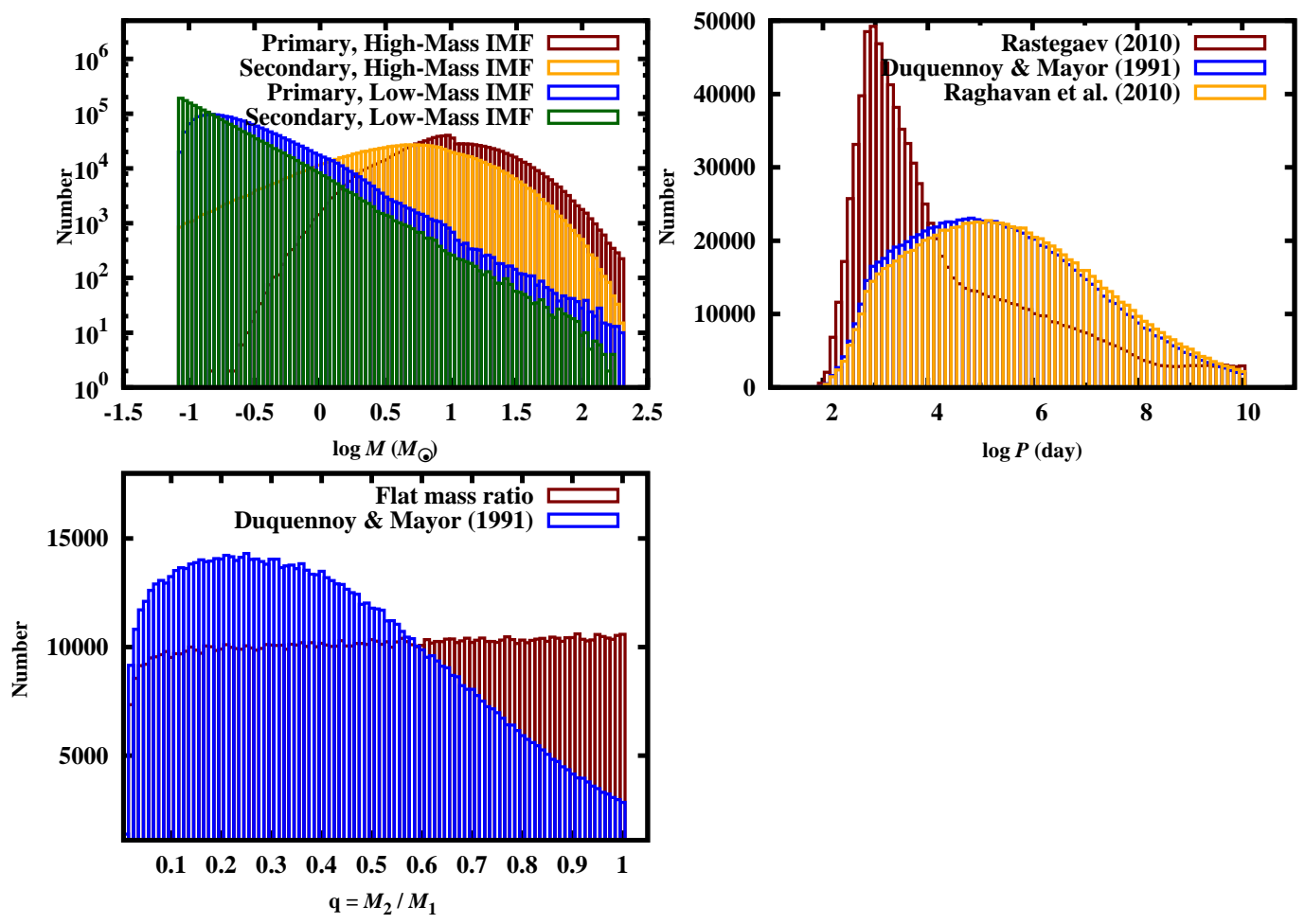

Figure 1: Demonstration of the simulation of binary systems. The total number of binary systems for given metallicity is set at one million for high-mass IMF and two millions for low-mass IMF in this figure. The distributions of the mass of primary and secondary stars are shown in the top left panel. The bottom left panel shows the distribution of the binary mass ratio. The top right panel shows the period distribution functions adopted in our simulations.

pendent of metallicity. The He-FDDM is assumed to occur for models with $0.8 \leq M / M_{\odot} \leq 3.5$ and with $[\mathrm{Fe} / \mathrm{H}] \leq-2.5$. We consider the effect of type 1.5 supernovae as a result of the suppression of mass loss at low-metallicity [3]. The boundary mass and metallicity for this event are set at $5 \leq M / M_{\odot} \leq 8$ and $[\mathrm{Fe} / \mathrm{H}] \leq-2.5$. A novel finding of our study is that a significant contribution from Type 1.5 supernovae is expected due to the suppression of mass loss from intermediate-mass AGB stars.

Models are compared with metal-poor giants in the SAGA database [17] and the SDSS sample [18]. We picked up comparison stars in the mass range of $0.82 \leq M / M_{\odot} \leq 0.83$ from our simulations that are representatives of currently surviving halo giants. The details of the models are described in a forthcoming paper (Suda et al., in prep.).

\section{Results and discussion}

Figures 2 shows the comparisons of the frequency of CEMP stars as a function of metallicity for different period distribution functions and for different adopted IMFs. The models show that the CEMP/EMP frequency increases with decreasing metallicity, in reasonable agreement with observations. 


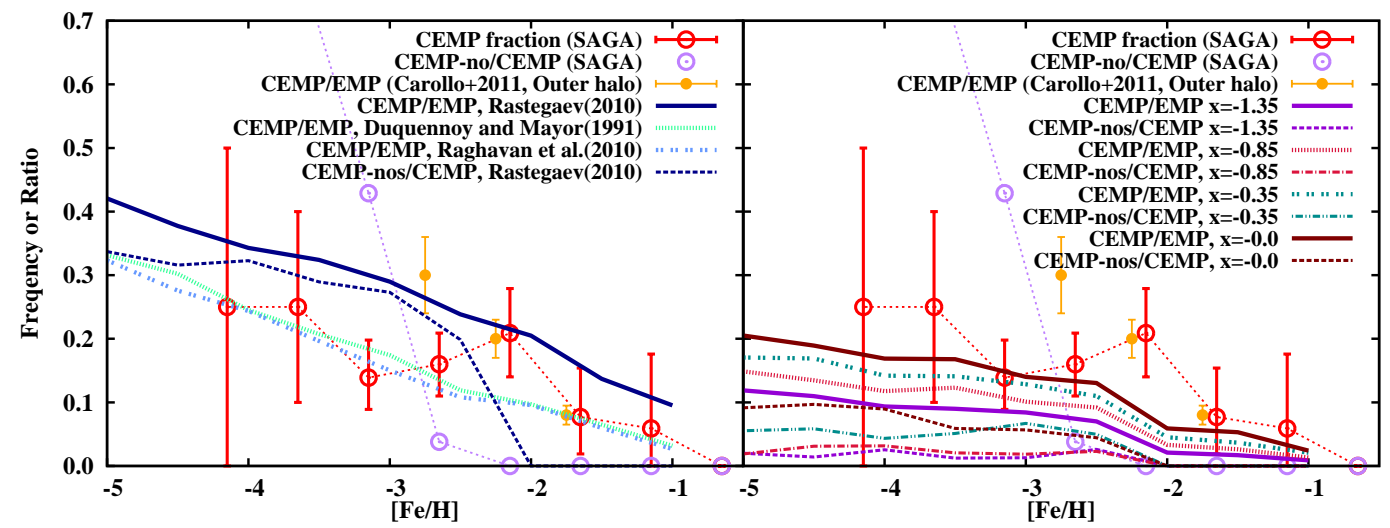

Figure 2: CEMP star frequency as a function of metallicity. Comparisons are made for different period distribution functions (left panel) and IMFs (right panel). The observed frequency of carbon-enhanced stars are taken from the SAGA database[17] and from the SDSS sample[18]. The error bars are estimated by bootstrap method. The fraction of CEMP-no to CEMP is also compared for EMP population.

The left panel of Figure 2 shows that our log-normal IMF is consistent with both the observed CEMP/EMP and CEMP-no/CEMP ratios at $[\mathrm{Fe} / \mathrm{H}] \leq-2$. This is due to the suppression of mass loss for intermediate-mass stars, which can reduce the number of NEMP stars. As seen in the right panel of Figure 2, CEMP/EMP ratio at $[\mathrm{Fe} / \mathrm{H}] \leq-2$ can be reproduced by a flat IMF except at $[\mathrm{Fe} / \mathrm{H}]=-2.5$, but the ratio of CEMP-no to CEMP disagrees with the observations. Our preliminary trial to find a solution that is consistent with observations, by changing parameters, turned out to be unsuccessful because the number of CEMP-no progenitors is too small to be consistent with observations. Our tentative conclusion is that, in any IMF we adopted, a transition of the IMF is required to account for the entire trend of CEMP frequency. This result supports the previous speculation by [19] that the IMF change should occur at $[\mathrm{Fe} / \mathrm{H}] \sim-2$.

For any choice of the IMF, mass loss at low-metallicity is a very important factor to determine the fraction of CEMP subclasses. More detailed discussions and robust conclusions will be given in a forthcoming paper (Suda et al., in prep.).

\section{Acknowledgments}

This work has been supported by Grant-in-Aid for Scientific Research (23224004), from Japan Society of the Promotion of Science (JSPS). T.S. acknowledges the support of the Institutional Program for Young Researchers Overseas Visits by JSPS, which enhanced the results and discussions in this work.

\section{References}

[1] T. C. Beers, and N. Christlieb 2005 The Discovery and Analysis of Very Metal-Poor Stars in the Galaxy, A\&AR 43, 531

[2] S. Lucatello et al. 2005 The Binary Frequency Among Carbon-enhanced, s-Process-rich, Metal-poor Stars, ApJ 625, 825

[3] P. R. Wood 2011 The Pulsation of Extremely Metal Poor AGB Stars, ASP Conf., 451, 87 
[4] Bromm, V. and Coppi, P. S. and Larson, R. B., 2002 The Formation of the First Stars. I. The Primordial Star-forming Cloud, ApJ 564, 23

[5] Y. Komiya, T. Suda, H. Minaguchi, T. Shigeyama, W. Aoki, and M. Y. Fujimoto 2007 The Origin of Carbon Enhancement and the Initial Mass Function of Extremely Metal-poor Stars in the Galactic Halo, ApJ 658, 367

[6] Komiya, Y. and Suda, T. and Fujimoto, M. Y., 2009 Early-Age Evolution of the Milky Way Related by Extremely Metal-Poor Stars, ApJ 694, 1577

[7] D. Raghavan et al. 2010 A Survey of Stellar Families: Multiplicity of Solar-type Stars, ApJS 190, 1

[8] A. Duquennoy, and M. Mayor 1991 Multiplicity among solar-type stars in the solar neighbourhood. II - Distribution of the orbital elements in an unbiased sample, A\&A 248, 485

[9] D. A. Rastegaev 2010 Multiplicity and Period Distribution of Population II Field Stars in Solar Vicinity, AJ 140, 2013

[10] T. Suda, and M. Y. Fujimoto 2010 Evolution of low- and intermediate-mass stars with [Fe/H] $\leq-2.5$, MNRAS 405, 177

[11] P. Gil-Pons, and C. L. Doherty 2010 The upper mass limit for the formation of TP-SAGB stars and the dredge-out phenomenon., Mem. S. A. It. 81, 974

[12] A. I. Karakas 2010 Updated stellar yields from asymptotic giant branch models, MNRAS 403, 1413

[13] J. R. Hurley, C. A. Tout, and O. R. Pols 2002 Evolution of binary stars and the effect of tides on binary populations, MNRAS 329, 897

[14] R. G. Izzard, E. Glebbeek, R. J. Stancliffe, and O. R. Pols 2009 Population synthesis of binary carbon-enhanced metal-poor stars, A\&A 508, 1359

[15] M. Y. Fujimoto, Y. Ikeda, and I. Iben, Jr. 2000 The Origin of Extremely Metal-poor Carbon Stars and the Search for Population III, ApJ 529, L25

[16] T. Suda, M. Aikawa, M. N. Machida, M. Y. Fujimoto, and I. Iben, Jr. 2004 Is HE 0107-5240 A Primordial Star? The Characteristics of Extremely Metal-Poor Carbon-Rich Stars, ApJ 611, 476

[17] T. Suda et al. 2008 Stellar Abundances for the Galactic Archaeology (SAGA) Database Compilation of the Characteristics of Known Extremely Metal-Poor Stars, PASJ 60, 1159

[18] D. Carollo et al. 2012 Carbon-enhanced Metal-poor Stars in the Inner and Outer Halo Components of the Milky Way, ApJ 744, 195

[19] T. Suda et al. 2011 The Stellar Abundances for Galactic Archaeology (SAGA) data base - II. Implications for mixing and nucleosynthesis in extremely metal-poor stars and chemical enrichment of the Galaxy, MNRAS 412, 843 